\title{
A Microwave Holographic Procedure for Large Symmetric Reflector Antennas Using a Fresnel-Zone Field Data Processing
}

\author{
Giuseppe Mazzarella, ${ }^{1}$ Giorgio Montisci, ${ }^{1}$ and Giampaolo Serra ${ }^{2}$ \\ ${ }^{1}$ Dipartimento di Ingegneria Elettrica ed Elettronica, Università di Cagliari, Piazza D’Armi, 09123 Cagliari, Italy \\ ${ }^{2}$ Osservatorio Astronomico di Cagliari, Istituto Nazionale di Astrofisica, Loc. Poggio dei Pini, 09012 Capoterra, Italy \\ Correspondence should be addressed to Giorgio Montisci, giorgiom@diee.unica.it
}

Received 28 September 2011; Accepted 19 December 2011

Academic Editor: Claudio Gennarelli

Copyright (C) 2012 Giuseppe Mazzarella et al. This is an open access article distributed under the Creative Commons Attribution License, which permits unrestricted use, distribution, and reproduction in any medium, provided the original work is properly cited.

In this paper we propose a new holographic procedure for the diagnostic of large reflector antennas, based on the direct use of the Fresnel-field pattern. The relation leading from the Fresnel field to the current on the reflector surface is formulated in the least-squares sense as a discrete data inverse problem and then regularized by using a singular value decomposition approach. A detailed theoretical analysis of the problem and full assessment of the presented technique are provided. Simulations are carried out by using the radiative near-field pattern generated with a commercial software. Results show good accuracy and robustness to noise for the retrieval of the panel-to-panel misalignment of a reflector antenna.

\section{Introduction}

The surface diagnostic of large reflector antennas, such as radio telescopes, is a key step in order to preserve their performance at all operating frequencies. The main reflector of a radio telescope is usually composed by a set of panels, which must be aligned to an ideal surface profile, with as the greater accuracy as possible.

The surface profile accuracy is mainly limited by two factors:

(i) the mechanical fabrication tolerance of the panels;

(ii) the alignment of the panels, which can be strongly modified by surface heating and gravitational load.

Therefore, in order to achieve the required antenna efficiency even at high operating frequencies, the primary reflector surface must be periodically checked. This means that the misalignments of the panels must be detected by using an effective and accurate diagnostic procedure and adjusted when it is possible.

Among the different measurement methods available in the open literature [1-7], we focus our attention on the microwave holography, an inverse method for the reflector surface profile reconstruction. This technique obtains the current induced on the reflector surface, and then the information about the surface misalignments, by the inversion of the antenna field pattern.

The measurement of the radiation pattern of large reflector antennas can be performed by pointing the antenna towards a radio source and changing the antenna azimuth and elevation along the points of a suitable grid. Nikolic et al. [8] use a cosmic radio source in order to perform measurements at different elevation angles and to check the reflector surface at high frequencies by using the radio astronomical receivers. In this case the signal-to-noise ratio (SNR) is enough to recover large-scale deformations, but not sufficient to retrieve the panel-to-panel misalignments with the same accuracy. Therefore, the signals of geostationary satellites [9] in the $\mathrm{X}$ and $\mathrm{Ku}$ band are commonly used as sources, since they provide a higher SNR than the cosmic sources.

If a source with the required SNR and operating frequency is not available in far field, a terrestrial transmitter in the radiative near field (known as Fresnel zone field) of the 
antenna under test (AUT) can be used for the holographic measurements [10]. In these cases, the reduced distance between the AUT and the transmitter allows a higher SNR than that achievable with far field measurements. Moreover, the operating frequency of the transmitter can be suitably chosen in order to arrange the measuring distance based on the morphology of the site around the AUT and/or to improve the accuracy of the deformation map.

The subject of this paper is microwave holography from Fresnel-field data. In Section 2 we will describe the issues related to this topic. In Sections 3 and 4 mathematical details of the procedure proposed in this paper have been discussed. Then, in Section 5, a number of surface deformations of arbitrary amount and position have been considered on a test axis symmetric parabolic reflector. These deformations have been recovered with good accuracy and the robustness to noise has been extensively checked.

A commercial software for the analysis of reflector antennas (GRASP 9 by TICRA) has been used to generate the Fresnel-field data of a parabolic reflector with a surface made up of a set of adjustable panels. The geometry of the reflector under test has been chosen equal to the $64 \mathrm{~m}$ primary reflector of the Sardinia Radio Telescope (SRT), a radio telescope with active surface, which will be soon inaugurated in Italy. The operating frequency has been selected at $22 \mathrm{GHz}$ based on the required surface accuracy.

\section{Holography from Fresnel Pattern}

The performance of large reflector antennas depends on the ability to keep the maximum surface accuracy even at high operating frequencies. Indeed, in order to achieve a high antenna aperture efficiency $\eta$, the surface deformation must be small compared to the free space wavelength $\lambda$, as shown in the Ruze formula [11]:

$$
\frac{\eta}{\eta_{0}}=\exp \left[-\left(\frac{4 \pi \varepsilon}{\lambda}\right)^{2}\right]
$$

wherein $\eta_{0}$ is the aperture efficiency of an ideal reflector and $\varepsilon$ is the RMS value of the deformation error on the actual reflector surface. For example, from (1), we derive that a surface efficiency of $67 \%$ can be achieved if the RMS error is less than $\lambda / 20$, that is, $680 \mu \mathrm{m}$ at $22 \mathrm{GHz}$ and $130 \mu \mathrm{m}$ at $115 \mathrm{GHz}$.

Therefore, in order to obtain a high efficiency at operating frequencies in the $\mathrm{K}$ band and over, the accuracy in the evaluation of the reflector surface must be of the order of a few hundred microns. As a consequence, in the holographic diagnostic procedure, the measurement frequency must be set in the K band or over, and a high SNR is needed to achieve the required reconstruction accuracy.

Microwave holography from Fresnel-field data provides a high SNR and flexibility in the choice of the measurement frequency. Nevertheless, the closeness between the terrestrial transmitter and the AUT limits the measurement elevation angles (usually below 10 degrees).

From the point of view of the data analysis, a critical aspect of this holographic method is that only a limited

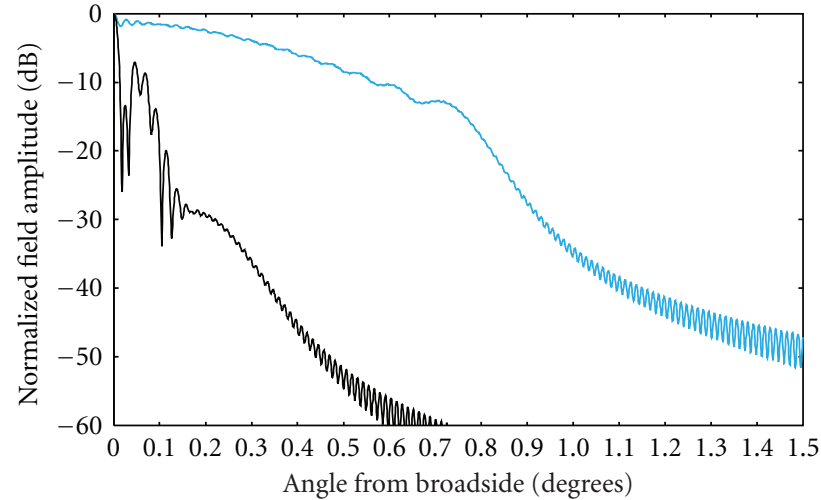

Figure 1: Comparison between simulated Near-Field pattern at the distance of $2160 \mathrm{~m}$ (blue line), and Near-Field pattern, at the distance of $2160 \mathrm{~m}$, with an axial displacement of the feed $\Delta f=$ $301 \mathrm{~mm}$ (black line).

amount of information on the antenna field is available to calculate the current induced on the reflector surface. In addition, a smooth Green's function and a limited integration domain complicate the computation of the surface current and ask for a regularized solution to an illconditioned inverse problem [12].

Recently, a holographic method for large reflector antennas, based on the use of Fresnel-field data, has been proposed by Baars et al. [10]. They apply a Fourier transform inversion [3] on data which have been suitably phase-distorted. In practice, this implies a refocusing of the parabolic reflector by means of an axial displacement of the feed. This displacement must be properly evaluated in order to minimize the rapid phase variation on the aperture plane, before the Fourier transform is applied.

The main consequence of this technique is the modification of the dynamic range of the measured field. As a matter of fact, the large longitudinal displacement of the feed (of the order of tens of wavelengths) defocuses significantly the far field while, at the same time, narrowing the Fresnel-field.

In Figure 1, we show the field calculated in the Fresnel zone, with and without an axial displacement of the feed $\Delta f$. The value $\Delta f=301 \mathrm{~mm}$ (used in Figure 1) has been calculated by applying the algorithm proposed by Baars et al. [10] to SRT at $22 \mathrm{GHz}$.

The approach of [10] provides a good approximation of the relationship between the Fresnel-field pattern and the aperture field, but the residual phase variation does not allow to make a rigorous inversion of this electromagnetic problem. Moreover, each AUT allows only a limited backand-forth shift of the feed along the reflector axis, which is set by its design and operative constraints. As a consequence, the axial displacement $\Delta f$, which minimizes the phase variation on the aperture plane, cannot be implemented for all AUTs.

In this paper we propose a microwave holographic technique, applied to axis symmetric reflectors, based on the direct use of the Fresnel zone field data. These data are not used to obtain the far field pattern by means of a NFFF transformation, as in [13], but they are directly used 


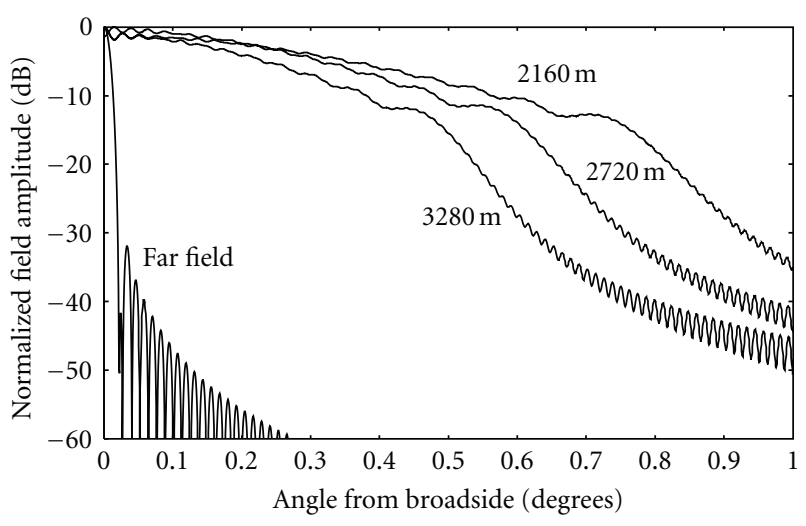

FIGURE 2: Simulated amplitude pattern at $22 \mathrm{GHz}$ of Sardinia Radio Telescope (64 $\mathrm{m}$ diameter parabolic reflector) for different distances.

by the procedure, without any correction, as in [10]. The relation between the Fresnel zone field and the current on the parabolic reflector surface is formulated in the leastsquares sense as a discrete data inverse problem and then regularized by using a singular value decomposition (SVD) approach. This procedure is based on the exact relationship between the field in the Fresnel zone and the current induced on the reflector surface, and it requires neither a paraxial approximation, as in the case of microwave holography from far field data [3], not the axial displacement of the feed [10].

Nevertheless, the use of the Fresnel pattern for microwave holographic diagnostic needs some important clarifications, as pointed out in the following discussion.

A typical sampling window in the far field of the AUT includes the main lobe and the few first side lobes. Therefore, its dimension is determined by the required surface resolution $[4,6]$. On the other hand, the dynamic range of the Fresnel pattern depends on the distance of the observation window and is always much smaller than the far field one (Figure 2). As a consequence, attention must be paid to the choice of the sampling window, which has to provide enough information to extract the reflector surface current. Actually, holography from near-field data requires a larger sampling window than holography from far field pattern to collect the same amount of information. Therefore, a larger number of samples must be measured. This improves the spatial resolution, but obviously increases the measurement time.

\section{Problem Framework}

The relationship between the field in the Fresnel zone [14] $\underline{E}(r, \theta, \phi)$ and the current $\underline{J}\left(\underline{r}^{\prime}\right)$ on the reflector surface is

$$
\begin{aligned}
\underline{E}(r, \theta, \phi)= & -j \beta \xi \frac{e^{-j \beta r}}{4 \pi r} \underline{F}(r, \theta, \phi), \\
\underline{F}(r, \theta, \phi)= & {\left[\underline{\underline{I}}-\underline{i}_{r} \underline{i_{r}}\right] } \\
& \cdot \iint_{S^{-}}\left(\underline{r}^{\prime}\right) e^{\left\{j \beta \underline{i}_{r} \cdot \underline{r}^{\prime}-j \beta(1 / 2 r)\left[\left(r^{\prime}\right)^{2}-\left(\underline{i}_{r} \cdot \underline{r}^{\prime}\right)^{2}\right]\right\}} d s,
\end{aligned}
$$

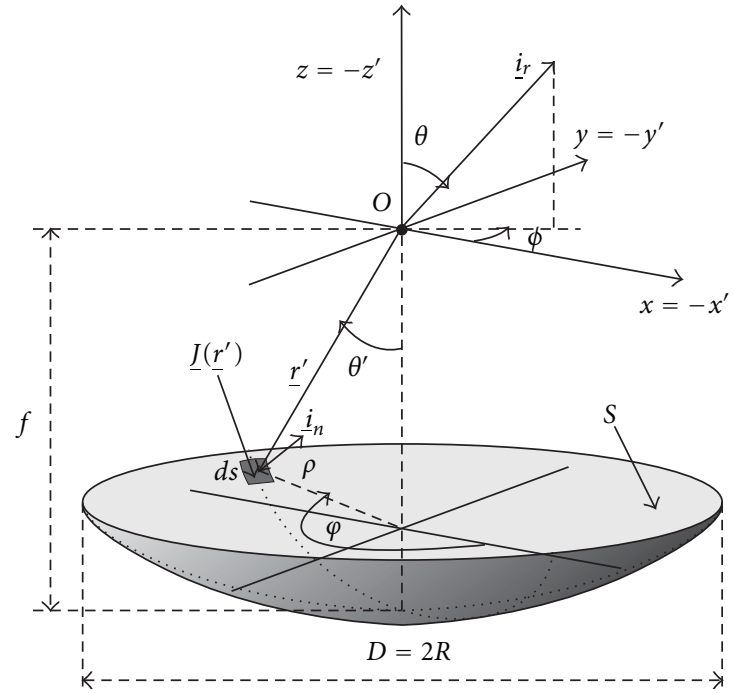

Figure 3: Reflector Geometry.

wherein $r$ is the distance of the measurement points, which is chosen constant for all the samples of the measurement window, $\beta=2 \pi / \lambda, \lambda$ being the free-space wavelength, $\xi=$ $120 \pi$ is the free-space impedance, $\underline{I}$ is the identity matrix, $\underline{i}_{r}$ is the unit vector that points toward the direction $(\theta, \phi)$, $S$ is the surface of the parabolic reflector (with diameter $D$ and focal length $f$ ), and $r^{\prime}$ is the vector that points on the reflector surface element $d s$ (see Figure 3 ).

The polarization of radio astronomical feeds is almost perfectly pure. Therefore for sake of simplicity, we assume that the reflector antenna is illuminated by a feed, located at the focal point ( $O$ in Figure 1), with an ideal linear polarization. Since the reflector is axis symmetric, we choose the $x$-axis along the $E$-plane of the feed.

The current induced on the reflector surface $J_{i}\left(\underline{r}^{\prime}\right)$ is our unknown. However, we can take advantage of the polarization purity of the feed to reformulate, and solve, the problem as a scalar one. We assume that the current direction $\underline{i}_{j}$ can be computed as the direction of the physical optics (PO) currents.

The direction of the incident magnetic field generated by the feed is

$$
\underline{i}_{H}=\frac{-\sin ^{2} \theta^{\prime}(\sin (2 \varphi) / 2) \underline{i}_{x}+(\mathfrak{N}) \underline{i}_{y}-\left(\sin \left(2 \theta^{\prime}\right) / 2\right) \sin \varphi \underline{i}_{z}}{\left|-\sin ^{2} \theta^{\prime}(\sin (2 \varphi) / 2) \underline{i}_{x}+(\mathfrak{N}) \underline{i}_{y}-\left(\sin \left(2 \theta^{\prime}\right) / 2\right) \sin \varphi \underline{i}_{z}\right|},
$$

where $\mathfrak{N}$ denotes $1-\sin ^{2} \theta^{\prime} \sin \varphi$, wherein $\theta^{\prime}(\rho)=$ $2 \arctan [\rho /(2 f)]$ is the angle measured from the feed boresight and $(\rho, \varphi)$ are the aperture coordinates $\left(z^{\prime}=-z, \quad x^{\prime}=\right.$ $\left.x=\rho \sin \phi, \quad y^{\prime}=-y=\rho \cos \phi\right)$ (see Figure 3).

Then, letting $\underline{i}_{n}$ the normal to the reflector surface, we assume

$$
\underline{i}_{j}=\frac{\underline{i}_{n} \times \underline{i}_{H}}{\left|\underline{i}_{n} \times \underline{i}_{H}\right|},
$$

so that the current amplitude $J\left(\underline{r}^{\prime}\right)$, defined as $\underline{J}_{i}\left(\underline{r}^{\prime}\right)=$ $J\left(\underline{r}^{\prime}\right) \underline{i}_{j}$, becomes the unknown of the problem. 
The integral (3) can be written in terms of the aperture coordinates $(\rho, \varphi)$ by using the surface Jacobian transformation [14]:

$$
\begin{aligned}
& \underline{F}(r, \theta, \phi) \\
& =\left[\underline{\underline{I}}-\underline{i}_{r} \underline{i}_{r}\right] \cdot \iint_{S^{\prime}} J(\rho, \varphi) \underline{i}_{j} e^{\left\{j \beta \underline{i}_{r} \cdot \underline{r}^{\prime}-j \beta(1 / 2 r)\left[\left(r^{\prime}\right)^{2}-\left(\underline{i}_{r} \cdot \underline{r}^{\prime}\right)^{2}\right]\right\}} B \rho d \rho d \varphi,
\end{aligned}
$$

wherein the Jacobian $B$ is given by

$$
B=\left[\left(\frac{\partial g\left(x^{\prime}, y^{\prime}\right)}{\partial x^{\prime}}\right)^{2}+\left(\frac{\partial g\left(x^{\prime}, y^{\prime}\right)}{\partial y^{\prime}}\right)^{2}+1\right]^{1 / 2}
$$

and $g\left(x^{\prime}, y^{\prime}\right)=z^{\prime}$ is the function describing the surface of the parabolic reflector.

The new integration domain $S^{\prime}$ in (6) is the projection of the reflector surface $S$ on the aperture plane and therefore is defined in the following range: $\rho \in\left[R_{b}, R\right], \varphi \in[0,2 \pi]$, where $R_{b}$ and $R$ are, respectively, the blocking and external radii of the reflector.

Let us now define an equivalent current $\widetilde{J}(\rho, \varphi)=$ $B J(\rho, \varphi)$ and replace $\underline{i}_{r}$ and $\underline{r}^{\prime}$ by their spherical components. Equation (6) becomes

$$
\begin{aligned}
\underline{F}(r, \theta, \phi)= & {\left[\underline{\underline{I}}-\underline{i}_{r} \underline{i}_{r}\right] } \\
& \cdot \int_{R_{b}}^{R} \int_{0}^{2 \pi} \underline{i}_{j} \tilde{J}(\rho, \varphi) G(r, \theta, \phi, \rho, \varphi) \rho d \rho d \varphi,
\end{aligned}
$$

where

$$
\begin{aligned}
& G(r, \theta, \phi, \rho, \varphi) \\
& =\exp \left[j \beta\left(\alpha \rho^{2}-2 \eta z^{\prime 2}\right)\right] \\
& \cdot \exp \left\{j \beta\left[z^{\prime} \cos \theta+\left(\rho \sin \theta+\rho z^{\prime} \gamma\right) \cos (\phi-\varphi)\right]\right\} \\
& \cdot \exp \left\{j \beta\left[\eta \rho^{2} \cos 2(\phi-\varphi)\right]\right\}, \\
& \alpha(r, \theta)=\frac{\sin ^{2} \theta-2}{4 r}, \\
& \eta(r, \theta)=\frac{\sin ^{2} \theta}{4 r}, \\
& \gamma(r, \theta)=\frac{\sin 2 \theta}{2 r},
\end{aligned}
$$

and $z^{\prime}(\rho)=\left[\rho^{2} /(4 f)\right]-f$ is the reflector nominal profile.

Now, since our attention is aimed at the reflector profile, that is, at a scalar quantity, only one component of the induced current is sufficient to obtain the required information on the reflector surface. As a consequence, only one component of the Fresnel field can be considered, and we choose the field copolar component

$$
\begin{aligned}
F_{c}(r, \theta, \phi)= & \underline{i}_{c} \cdot\left[\underline{\underline{I}}-\underline{i}_{r} \underline{\underline{i}}_{r}\right] \\
& \cdot \int_{R_{b}}^{R} \int_{0}^{2 \pi} \underline{i}_{j} \tilde{J}(\rho, \varphi) G(r, \theta, \phi, \rho, \varphi) \rho d \rho d \varphi,
\end{aligned}
$$

because it allows for the best SNR on the data. In (10) the vector

$$
\begin{aligned}
\underline{i}_{c}= & \cos \phi \underline{i}_{\theta}-\sin \phi \underline{i}_{\varphi} \\
= & {\left[1-\cos ^{2} \phi(1-\cos \theta)\right] \underline{i}_{x} } \\
& -(1-\cos \theta) \sin \phi \cos \phi \underline{i}_{y}-\sin \theta \cos \phi \underline{i}_{z},
\end{aligned}
$$

is the field copolar component direction (Ludwig's third definition [15]), assuming that the radiated electric field is predominantly $x$-polarized. Note that, for large and focusing reflectors, as radio astronomical ones, we can assume $\theta \ll 1$ ( $\cos \theta \cong 1$ and $\sin \theta \cong 0$ ) and find an approximate expression for $(11)$

$$
\underline{i}_{c} \cong \underline{i}_{x}
$$

It is worth noting that no paraxial approximation has been made in the phase terms of (10). As a matter of fact, we used $\theta \ll 1$, to approximate only the amplitude and direction of the copolar field component, as apparent from (12).

Equation (10) is a (two-dimensional) linear Fredholm integral equation of the first kind [12], where $\tilde{J}(\rho, \varphi)$ is the unknown and $F_{c}(\theta, \phi)$ the known term. Its inversion is a severely ill-posed problem because of its very smooth kernel and the limited integration domain $S^{\prime}$ [12]. It follows that (10) cannot be simply inverted, but it is necessary to apply a regularization procedure, which generally needs also a different solution definition, in view of the fact that the measured data $F_{c}(\theta, \phi)$ will include an unavoidable noise. Therefore, the problem must be analyzed as a discrete data inverse problem $[16,17]$.

Let $\left(\theta_{p}, \phi_{p}\right)$ be the set of field measurement points (with $p=1, \ldots, M$ ) and $L_{p}$ the functional which supplies $F_{c}\left(\theta_{p}, \phi_{p}\right)$. If $f_{p}$ are the measured values, then

$$
f_{p}=L_{p}[\tilde{J}]+n_{p}=f_{p}^{t}+n_{p},
$$

where $f_{p}^{t}$ is the field's "ideal" value (i.e., without noise) and $n_{p}$ is the noise on the $p$ th measure. In order to solve (13), the unknown current must be discretized too, by choosing a suitable set of basis functions $I_{q}(\rho, \varphi), q=1, \ldots, N$. Therefore, the unknown current can be expressed as $\tilde{J}=$ $\sum_{q=1}^{N} J_{q} I_{q}(\rho, \varphi)$, and (13) can be rewritten as

$$
f_{p}=\sum_{q=1}^{N} J_{q} L_{p}\left[I_{q}(\rho, \varphi)\right]+n_{p} .
$$

If the functional $L_{p}$ is not directly invertible, as in (10), the problem must be formulated in the least-squares sense. Defining the numerical vector $f \in \mathbb{C}^{M \times 1}$, having components $f_{p}$, and the matrix $\underline{\underline{L}}$ associated with the functional $L_{p}$, we must seek for a solution $\tilde{J}_{L S}$ (having components $J_{q}$ ) that minimizes the residual 2-norm

$$
\min _{\tilde{\tilde{I}} \in \mathbb{C}^{N \times 1}}\|\underline{\underline{L}} \cdot \underline{\tilde{J}}-\underline{f}\|_{2}^{2}
$$


This solution is usually termed "least-squares solution" or "pseudosolution" [12] and depends linearly on the data $f$, so that we can write

$$
\underline{\tilde{J}}^{L S}=\underline{\underline{L}}^{\dagger} \cdot \underline{f}
$$

wherein $\underline{L}^{\dagger}$ is the so-called generalized inverse (or MoorePenrose inverse) of the matrix $\underline{\underline{ }}$ [12]. The main point is that, even though this discrete data problem is well posed, it turns out to be heavily ill conditioned [12], because (10) is ill-posed. Therefore, it needs to be solved through a suitable regularization procedure [18].

\section{Solution Approach}

In Section 4.1 we describe the main steps that lead from the acquisition of the Fresnel-field data to the final map of the reflector surface. These steps will be discussed in detail in Section 4.2.

4.1. A Brief Overview of the Algorithm. The holographic procedure presented in this paper can be applied to reflector antennas whose external boundary is a circumference located on a plane orthogonal to the reflector axis. This assumption is mandatory for the examples presented in this paper. In fact, in order to reduce the computational load, we represent both the field and the current induced on the reflector surface as a truncated Fourier series. As a consequence, our procedure requires the following steps.

(i) The measurement points must be located on concentric circles (antenna framework), while typical measured data are available in azimuth-elevation coordinates. Therefore, the first operative step is a data transformation from the terrestrial framework (a regular lattice in azimuth elevation) to the antenna framework. This has been done using an interpolation with band-limited self-truncating functions [19].

(ii) Exploiting the representation of the field and the induced current as Fourier harmonics, we express the relation between the field and the current through a number of decoupled integral equations, one for each harmonic.

(iii) Each integral equation is transformed into a linear system. In order to do that: (a) the coefficients of the Fourier harmonics are expressed in terms of a set of piecewise-constant basis functions with respect to the radial coordinate; (b) the integral equation is sampled at the measurement points.

(iv) Each linear system is solved in the least-squares sense, by using a regularization procedure based on the singular value decomposition. The known term is the harmonic of the field sampled at the measurement points, and the unknown is the harmonic of the current discretized over the reflector surface, with respect to the radial coordinate. (v) After solving the linear systems, we obtain the Fourier harmonics of the induced current, and the truncated Fourier series gives the total induced current on the reflector surface.

(vi) Finally, the phase of the current induced on the displaced surface is compared to the phase of the current induced on the reference surface profile, after a suitable unwrapping procedure.

4.2. Description of the Holographic Procedure. The unknown current $\tilde{J}(\rho, \varphi)$ on the reflector surface is retrieved by using a formulation like (16). Therefore, $\tilde{J}(\rho, \varphi)$ is discretized according to the required graphical resolution, and the leastsquares solution is regularized by using the SVD.

The SVD approach applied to the calculation of a large linear system, as that deriving from the application of (16) to a large reflector antenna, is computationally heavy. However, this problem can be overcome by using the axial symmetry of a lot of radio telescopes. In fact, this feature allows to better represent both the surface current and the radiation pattern as Fourier series. Obviously, for numerical computation, it is necessary to truncate the series to a finite number of terms with suitable upper limits. These limits can be determined by exploiting the fact that the radiation pattern is a quasiband-limited function [20]. This property suggests that the number of the retained series terms can be obtained by the spatial bandwidth of the scattered field, as widely discussed in [20]. For a large scattering system, the effective bandwidth of the scattered field, measured on a circular domain, can be identified as

$$
w=\frac{2 P+1}{2 \sin \theta_{\max }}=\chi \beta R,
$$

wherein $R$ is the radius of the sphere which includes the scatterer (we assume it equal to the radius of the reflector); $\chi$ is an excess bandwidth factor and has to be set slightly larger than unity to ensure a negligible representation error $[19,20] ; \theta_{\max }$ is the maximum value of the $\theta$ angle of the measured circular domain (see Figure 3), $2 P+1$ is the number of the Fourier harmonics. Solving (17) with respect to $P$, it follows that the higher harmonic $P$ is given by

$$
P \cong \chi \beta R \sin \theta_{\max } .
$$

Therefore, letting $C_{i}(\rho)$ the $i$ th harmonic of the current and $t_{i}(\theta)$ the $i$ th harmonic of the field, the truncated Fourier series representation for the current and the radiation pattern is

$$
\begin{aligned}
& \tilde{J}(\rho, \varphi)=\sum_{i=-P}^{P} C_{i}(\rho) \exp [j(i \varphi)], \\
& F_{c}(\theta, \phi)=\sum_{i=-P}^{P} t_{i}(\theta) \exp [j(i \phi)],
\end{aligned}
$$

wherein each harmonic $t_{i}(\theta)$ can be calculated as

$$
2 \pi t_{i}(\theta)=\int_{0}^{2 \pi} F_{c}(\theta, \phi) \exp [-j(i \phi)] d \phi .
$$


Now, carrying out the scalar products in (10), substituting the expressions (19) and (21) in (10) and using the approximated expression (12), after calculating the integrals with respect to the variables $\varphi$ and $\phi$, we get a set of $2 P+1$ one-dimensional integral equations, one for each harmonic

$$
\begin{aligned}
t_{i}(\theta) \cong & \pi \int_{R_{b}}^{R}\left[4 \cos \left(\frac{\theta^{\prime}}{2}\right)-2 \sin \theta^{\prime} \sin \left(\frac{\theta^{\prime}}{2}\right)\right] Q_{i}(\rho, \theta) C_{i}(\rho) \rho d \rho \\
& +\frac{\pi}{4} \int_{R_{b}}^{R} \sin \theta^{\prime} \sin \left(\frac{\theta^{\prime}}{2}\right) Q_{i}(\rho, \theta) C_{i-2}(\rho) \rho d \rho \\
& +\frac{\pi}{4} \int_{R_{b}}^{R} \sin \theta^{\prime} \sin \left(\frac{\theta^{\prime}}{2}\right) Q_{i}(\rho, \theta) C_{i+2}(\rho) \rho d \rho
\end{aligned}
$$

with $\theta^{\prime}(\rho)=2 \arctan [\rho /(2 f)]$ and

$$
\begin{aligned}
Q_{i}(\rho, \theta)= & \exp \left[j \beta\left(\alpha \rho^{2}-2 \eta z^{\prime 2}+z^{\prime} \cos \theta\right)\right] \\
& \cdot \sum_{k=-\infty}^{\infty} j^{i-k} J_{i-2 k}\left(\beta \rho \sin \theta+\beta \rho z^{\prime} \gamma\right) J_{k}\left(\beta \eta \rho^{2}\right),
\end{aligned}
$$

wherein $J_{i}$ is the Bessel function of the first kind.

Based on the same consideration carried on in [18] we can neglect the coupling between the $i$ th harmonic of the field $t_{i}(\theta)$ and the $(i-2)$ th and $(i+2)$ th harmonics of the current $\left(C_{i-2}\right.$ and $\left.C_{i+2}\right)$. Therefore, we are left with a new set of $2 P+1$ decoupled equations

$$
\begin{array}{r}
t_{i}(\theta) \cong 4 \pi \int_{R_{b}}^{R} \cos \left(\frac{\theta^{\prime}(\rho)}{2}\right) Q_{i}(\rho, \theta) C_{i}(\rho) \rho d \rho \\
i \in[-P, P] .
\end{array}
$$

Each Fredholm equation (24) can be transformed into a linear system by representing the coefficient of the $i$ th Fourier harmonic $C_{i}(\rho)$ in terms of a set of piecewise-constant basis functions $\left\{I_{q}(\rho)\right\}, q=1, \ldots, N$. Therefore, we divide the integration domain of (24) into $N$ subdomains such that

$$
\Delta \rho=\frac{R-R_{b}}{N} .
$$

Then, we sample both sides of (24) at the measurement points.

This is not a direct task, since $F_{c}(\theta, \phi)$ (and then $t_{i}(\theta)$ ) requires the measurement points to be located on concentric circles (antenna framework), while typical measured data are available in azimuth-elevation coordinates $\left(\Theta_{h}, \Phi_{k}\right)$ (terrestrial framework). Therefore, a data transformation is required and can be performed in two steps.

(i) The measured data are transformed from the terrestrial framework (a regular lattice in azimuthelevation) to the antenna framework, which can be considered a regular lattice in $(u, v)$ for small $\theta$ angles (wherein $u=\sin \theta \cos \phi, v=\sin \theta \sin \phi$ );

(ii) The new data in the antenna framework are interpolated in a set of points placed on concentric circles with equispaced values $\theta_{p}=p \Delta \theta$, with $p=1, \ldots, M$ and $\Delta \theta=\theta_{\max } / M$. This interpolation is performed by using band-limited self-truncating functions [19], and, for each value of $\theta_{p}$, the number of interpolating points with respect to the $\phi$ coordinate, $n_{\phi}$, is determined by the constraint on the limited spatial band of the reflector field [20], that is, $n_{\phi}\left(\theta_{p}\right)=$ $\chi \beta R \sin \theta_{p}$. Therefore, the spacing between the field samples along each circle $\theta_{p}$ is $\Delta \phi_{p}=2 \pi /\left[2 n_{\phi}\left(\theta_{p}\right)+\right.$ $1]$.

The number of unknowns involved in the least-squares problems is determined by the choice of $N$, as apparent from (25). Nevertheless, for each harmonic we have a different linear system, with different matrix and data vector. More precisely, a given circular ring, at a distance $R_{0}$ from the reflector axis (i.e., all the reflector points having radial coordinate $\rho \cong R_{0}$ ), produces, in the measurements region, only field harmonics up to $P\left(R_{0}\right)=\hat{P}\left(R_{0}\right)=\chi \beta R_{0} \sin \theta_{\max }$ (see (18)). Therefore, its current must be retrieved by using only the harmonics in the range $\left[-\widehat{P}\left(R_{0}\right), \widehat{P}\left(R_{0}\right)\right]$. As a consequence, for the $i$ th harmonic, only $\tilde{N}_{i} \leq N$ unknowns are involved in the $i$ th linear system.

Furthermore, for each measurement point $\theta_{p}$, the limited spatial bandwidth of the scattered field is $(2 P+1) /\left(2 \sin \theta_{p}\right)=$ $\chi \beta R$ (see (17)). This means that the maximum index of the Fourier harmonics $P\left(\theta_{p}\right)$ is given by $P\left(\theta_{p}\right)=\chi \beta R \sin \theta_{p}$. As a consequence, the number of equations $\widetilde{M}_{i} \leq M$ of the $i$ th linear system is a decreasing function of $i$.

Then, we represent each harmonic $C_{i}(\rho)$ in terms of the set $\left\{I_{q}(\rho)\right\}$

$$
C_{i}(\rho)=\sum_{q=N-\tilde{N}_{i}+1}^{N} A_{q}^{(i)} I_{q}(\rho),
$$

and let $\underline{A}^{(i)}$ be the vector with components $A_{q}^{(i)}$ and $\underline{T}^{(i)}$ the vector with components $t_{i}\left(\theta_{p}\right)$, with $p=M-\widetilde{M}_{i}+1, \ldots, M$.

Since the solution of each $i$ th linear system can be found in the same way and independently from the others, we need a solution technique for the general form of such systems

$$
\underline{\underline{L}} \cdot \underline{A}=\underline{T}
$$

wherein $\underline{\underline{L}} \in C^{\widetilde{M} \times \tilde{N}}, \underline{A} \in C^{\tilde{N} \times 1}, \underline{T} \in C^{\widetilde{M} \times 1}$.

Equation (27) can be solved in the least-squares sense [12], by using a regularization procedure based on the SVD of the matrix $\underline{\underline{L}}$. In [18] the same method has been applied by the authors to the reflector surface retrieval problem, but using far field instead of Fresnel-field data. Therefore, the reader is referred to Section 4 of [18] for the details of this SVD regularization procedure. 


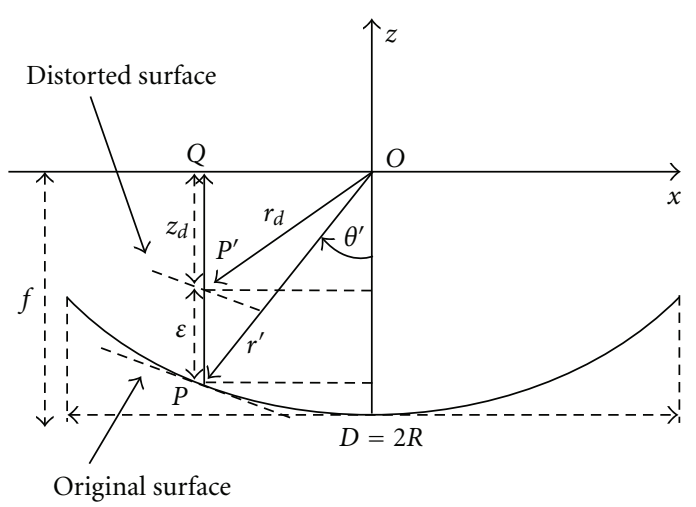

Figure 4: Description of surface deformation.

Once the least-squares solution $\underline{A}_{L S}^{(i)}$ has been computed, we can find the currents on the reflector as the sum of Fourier harmonics:

$$
\begin{aligned}
J_{\mathrm{LS}}(\rho, \varphi) & =\sum_{i=-P}^{P} C_{i}(\rho) \exp [j(i \varphi)] \\
& =\sum_{i=-P_{q}}^{P} \sum_{N-\tilde{N}_{i}+1}^{N} A_{q}^{(i)} I_{q}(\rho) \exp [j(i \varphi)],
\end{aligned}
$$

where the discretization of radial coordinate $\rho$ depends on the sampling points of the $J_{\mathrm{LS}}(\rho, \varphi)$ according to (25), whereas the angular coordinate $\varphi$ is suitably discretized according to the required graphical resolution.

The currents induced on the reflector surface (28) are related to the surface profile, and the comparison between the calculated profile and the nominal profile allows us to find the deformations of the reflector surface. In order to extract the surface profile information from (28), we assume that the phase center of the feed is placed at the focal point and that the surface error is described by the function $\varepsilon(x, y)$ in the $z$ direction, as shown in Figure 4. After simple geometrical considerations, the surface deformation is obtained by

$$
\varepsilon(x, y)=\frac{\lambda}{2 \pi\left(1+\cos \theta^{\prime}\right)} \Delta \delta
$$

wherein $\Delta \delta=\operatorname{phase}\left(J_{\text {ref }}\right)-\operatorname{phase}\left(J^{\mathrm{LS}}\right)$ is the difference between the phase of the reference current (induced on a reflector surface without deformations) and the phase of the recovered current. Both phases have been unwrapped by using the 2D procedure described in [21].

It is worth noting that the choice of a deformation in the $z$ direction has been made for compatibility with the deformations produced by the commercial software used to test our procedure (GRASP 9). Of course, the results can be easily extended to the case of a deformation in the direction normal to the surface profile with similar geometrical considerations. In any case, this choice depends only on the construction details of the active surface.

\section{Results and Discussion}

In order to assess the proposed technique, many different tests have been performed on a reflector with the same geometrical configuration of the SRT primary reflector. The reflector diameter is $64 \mathrm{~m}$, the blockage region diameter is $8 \mathrm{~m}$; and the focal length-diameter ratio is 0.33 . For the sake of simplicity, in our tests, we consider an ideal Gaussian feed, linearly polarized, with an aperture taper of $-12 \mathrm{~dB}$. Moreover, we suppose to neglect the diffraction effects due to the subreflector and the quadripod.

The reflector field in the Fresnel region has been simulated with GRASP, at the operating frequency of $22 \mathrm{GHz}$, on a suitable grid in the terrestrial framework, and then interpolated in the antenna framework using the selftruncating functions. The reflector model, created with GRASP, subdivides the reflector surface in a number of panels (Figures 5 and 8), whose dimensions and possible displacements with respect to the ideal profile can be assigned arbitrarily. In our case the number and the location of the panels have been chosen the same as SRT, that is, 1008 individual panels (with area in the range $\left[2.4 \mathrm{~m}^{2}-5.3 \mathrm{~m}^{2}\right]$ ), divided in 14 concentric circles (Figure 8).

In the GRASP modelling, each panel has up to four control points $\left(P_{1}, P_{2}, P_{3}\right.$, and $\left.P_{4}\right)$, that is, the position of the panel vertexes (Figure 5). For the sake of simplicity, we impose the panel misalignment by modifying only the $z$ coordinate of each control point of the same amount $\varepsilon$ for all the control points.

First of all, the phase of $J_{\text {ref }}$ (i.e., the surface current in the reference case) is evaluated starting from the reference field, that is, the Fresnel pattern generated by the nominal reflector, without any surface deformation. Then, a Fresnel pattern is generated starting from a surface profile with a test deformation obtained with a panel displacement in the $z$ direction. Finally, we get the estimation of this surface deformation by applying the solution approach described in Section 4 to retrieve both $J_{\text {ref }}$ and $J_{\text {LS }}$ and by using (29).

In Section 5.1 of this section we will discuss the issues related to the sampling of the field in the Fresnel region. In Section 5.2 we will apply our procedure to the reconstruction of the surface profile of the parabolic reflector under test.

5.1. The Choice of the Sampling Window. The inversion of the antenna field pattern requires that the measurement window provides enough information to derive the reflector surface current. This task is implicit in the holographic procedure from far field data, since far field pattern has a high dynamic range (Figure 2). Nevertheless, due to the reduced dynamic range of the near-field pattern, microwave holography from Fresnel data requires an accurate evaluation of the dimension of the sampling window, which must be large enough to contain the information needed to provide the reflector surface current with good accuracy. We evaluate the amount of information contained in the observation window by using the percentage of radiated power inside this window.

In order to quantify this problem we have created, by using the commercial software GRASP, the model of the prime reflector of SRT, including 1008 panels. We have 


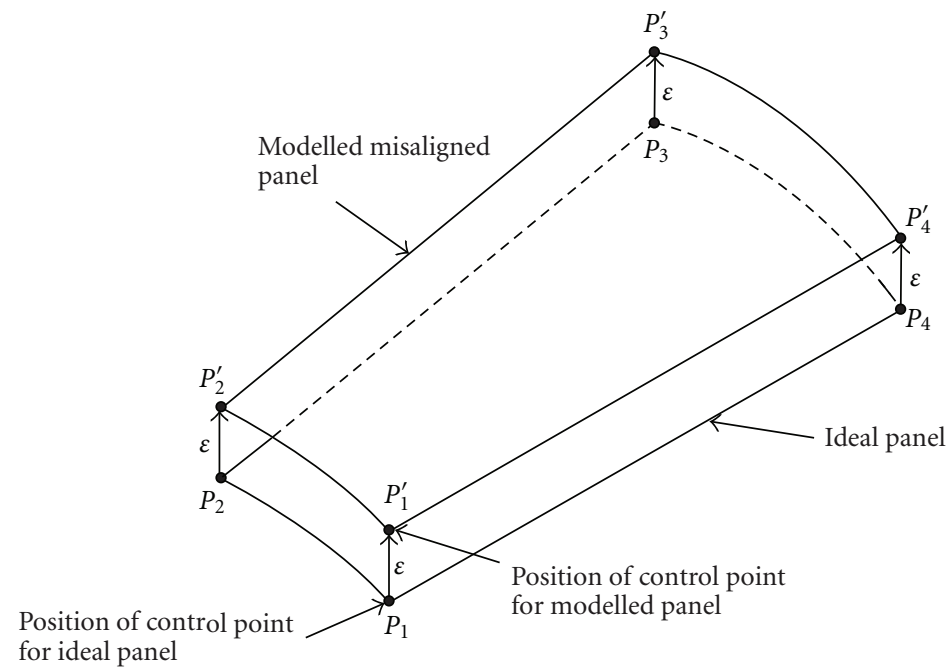

FIGURE 5: GRASP CAD modelization of a panel.

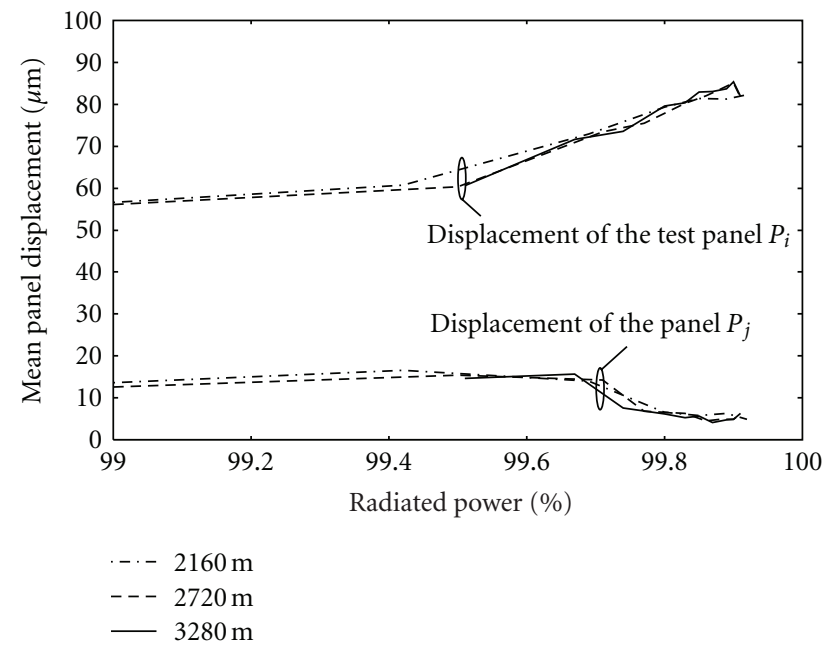

Figure 6: Deformation of $100 \mu \mathrm{m}$ of the panel under test $P_{i} . P_{j}$ (with $j \neq i$ ) is the panel with the highest mean deformation.

imposed a displacement of $100 \mu \mathrm{m}$ of a single panel $P_{i}$ identified by the following coordinates $\rho \in[26.24455 \mathrm{~m}$, $28.03525 \mathrm{~m}], \varphi \in\left[26.25^{\circ}, 30^{\circ}\right]$, whereas all the other panels are aligned to the ideal parabolic profile. Then, the proposed diagnostic procedure has been applied to the reconstruction of this surface profile.

The accuracy of the surface recovering technique has been evaluated by the comparison between the recovered deformation of the test panel $P_{i}$, which should be as near to $100 \mu \mathrm{m}$ as possible, and the recovered deformation of the rest of the reflector, which should be negligible if the diagnostic procedure works well.

Therefore, we let $P_{j}$ (with $j \neq i$ ) be the panel with the highest mean deformation among all the other panels of the reflector surface different from $P_{i}$. It is evident that the lower

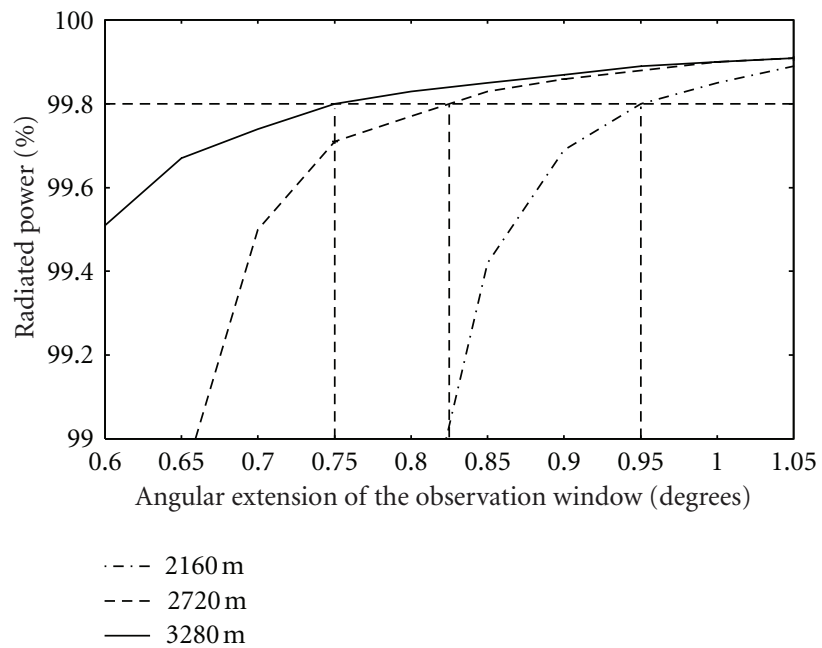

FIGURE 7: Power radiated by a parabolic reflector with the same geometry of SRT, as a function of the angular extension of the observation window.

the deformation of $P_{j}$, the lower the error in the recovered deformation of the panels different from $P_{i}$.

In Figure 6 we give a quantitative estimation of the accuracy of the technique for different percentage of the radiated power inside the observation window. We compare the deformation of the test panel $P_{i}$ and the deformation of the panel $P_{j}$. As apparent from this figure, the recovered displacement on the reflector panels depends only on the percentage of radiated power, independently of the distance of the terrestrial source, and good results are achieved when the power radiated inside the observation window is more than $99.8 \%$.

In Figure 7 the percentage of the radiated power as a function of the extension of the observation window is shown for different measuring distances. The test distances, 

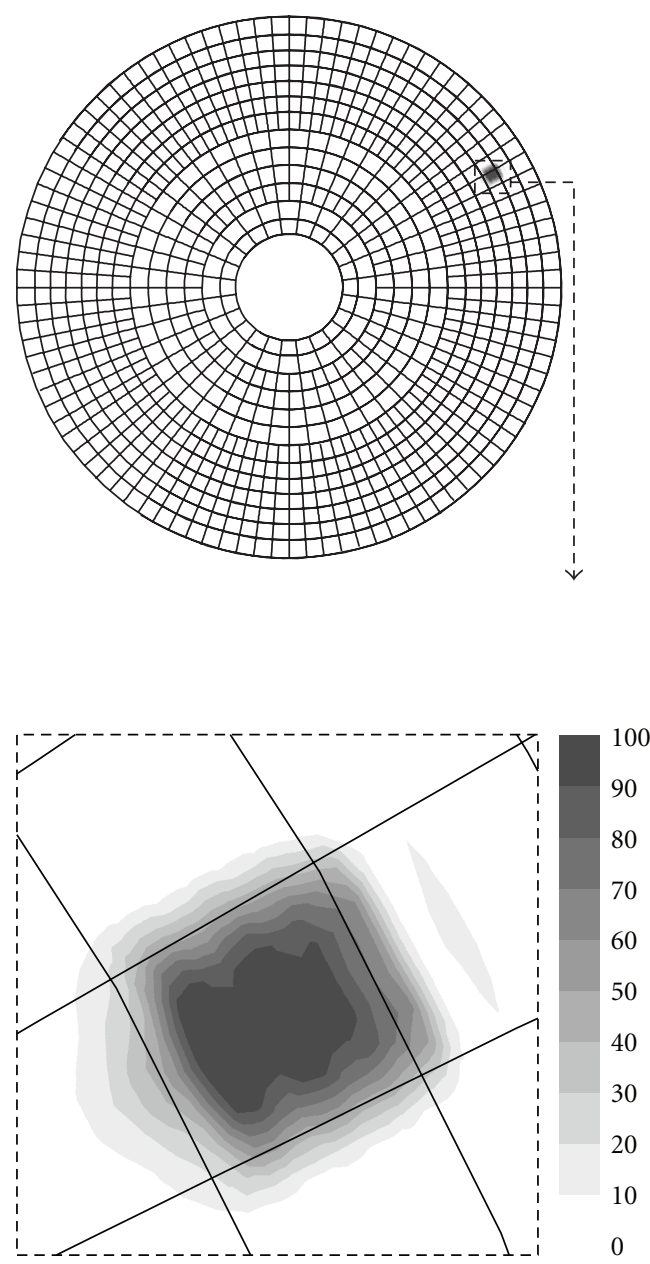

FIGURE 8: Gray scale map of the retrieved reflector surface for a deformation of $100 \mu \mathrm{m}$ of the panel under test. The gray scale bar is in unit of $\mu \mathrm{m}$.

$2160 \mathrm{~m}, 2720 \mathrm{~m}$, and $3280 \mathrm{~m}$, are all within the Fresnel zone. The angular extension of the sampling window, required to guarantee $99.8 \%$ of radiated power, can be calculated by using the curves in Figure 7.

5.2. Validation of the Holographic Procedure. The field data used to validate the holographic method proposed in this paper have been calculated by using the software GRASP 9 by TICRA, at a distance equal to $2160 \mathrm{~m}$. This choice would allow us to mount a radio transmitter over a tower placed on a nearby hill at $2160 \mathrm{~m}$ from the SRT. In this way, we could perform measurements at an elevation angle of about 8 degrees. We will not give any other details of a possible measurement scenario because this is not the aim of the present work.

Once the distance of the transmitter has been selected, both the frequency and the observation window are chosen according to Section 5.1.

Therefore, at $2160 \mathrm{~m}$, with an operating frequency of $22 \mathrm{GHz}$, the angular extension of the sampling window,

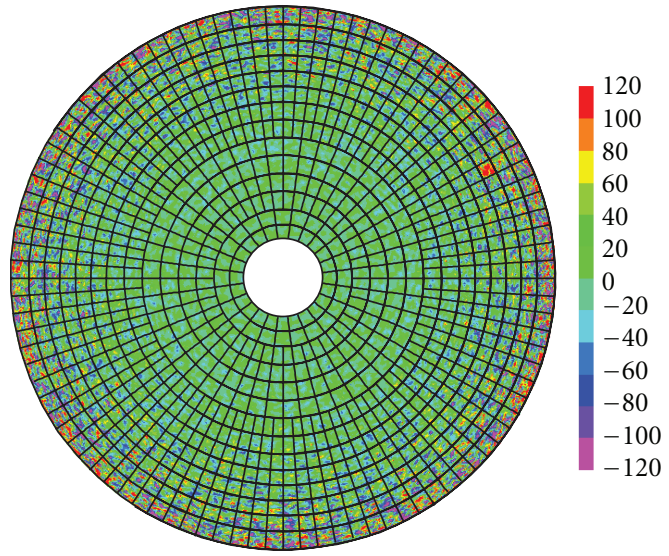

FIGURE 9: Map of the retrieved reflector surface for a deformation of $100 \mu \mathrm{m}$ of the panel under test. SNR $=40 \mathrm{~dB}$. The color bar is in unit of $\mu \mathrm{m}$.

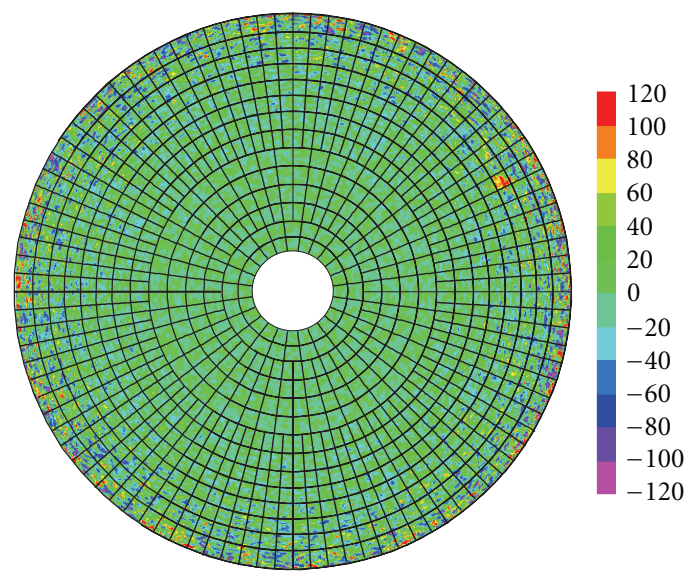

FIGURE 10: Map of the retrieved reflector surface for a deformation of $100 \mu \mathrm{m}$ of the panel under test. SNR $=44 \mathrm{~dB}$. The color bar is in unit of $\mu \mathrm{m}$.

corresponding to $99.8 \%$ of radiated power, is $\theta_{\max }=0.95 \mathrm{de}$ grees (Figure 7). Then $u_{\max }=v_{\max }=\sin \left(\theta_{\max }\right)=0.016581$ are the boundaries of the lattice in the antenna framework (coordinates $u, v$ ). According to the sampling theorem the number of sampling points in the antenna framework is $\left(N_{s}\right)^{2}$ with [3]:

$$
N_{s}=\operatorname{int}\left[2 u_{\max } \frac{D}{\lambda} \chi\right]+1 \text {, }
$$

wherein $D$ is the parabolic reflector diameter, $\lambda$ is the free space wavelength, and $\chi>1$ is an excess bandwidth factor, also called oversampling factor.

For the SRT geometry $(D=64 \mathrm{~m})$, with $\chi=1.203,(30)$ provides $N_{s}=188$.

As a consistency test, we have first recovered a displacement of $100 \mu \mathrm{m}$ of the panel under test $P_{i}$ by inversion of noise-free field data. This test has been fully successful, and in Figure 8 we show the greyscale map of the reflector with the recovered deformation. 


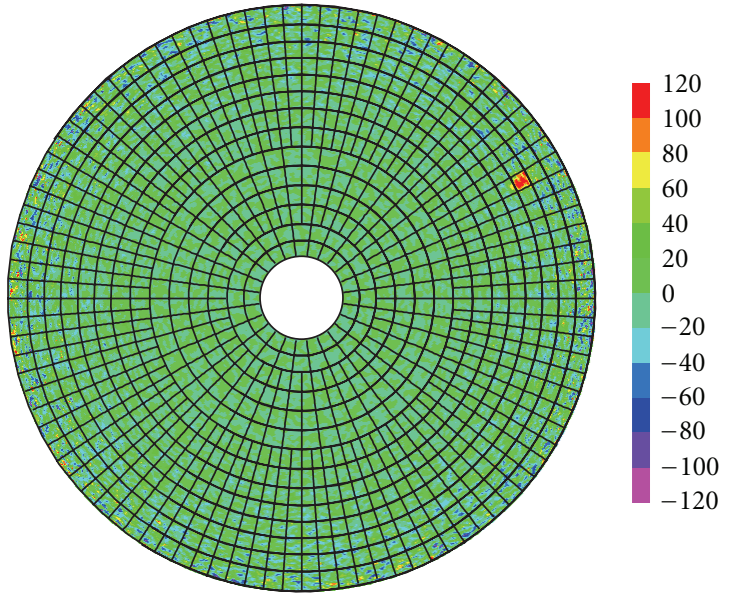

FIGURE 11: Map of the retrieved reflector surface for a deformation of $100 \mu \mathrm{m}$ of the panel under test. SNR $=48 \mathrm{~dB}$. The color bar is in unit of $\mu \mathrm{m}$.

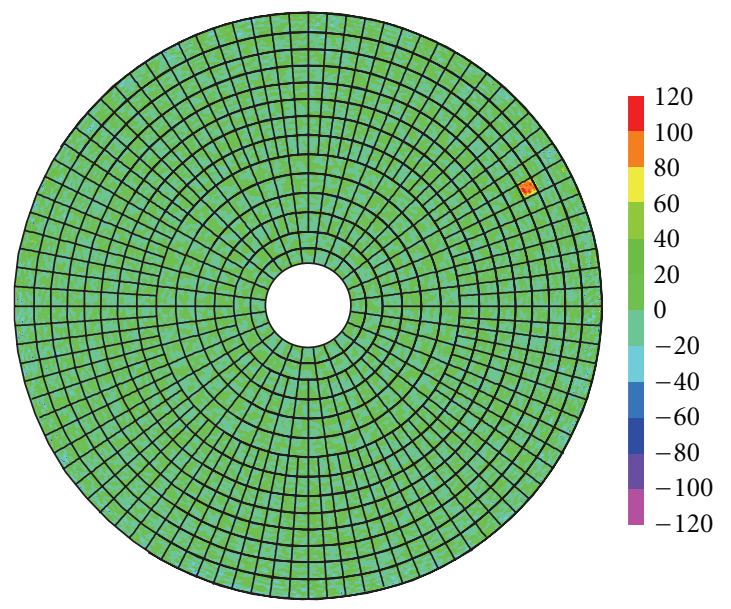

Figure 12: Map of the retrieved reflector surface for a deformation of $100 \mu \mathrm{m}$ of the panel under test. SNR $=56 \mathrm{~dB}$. The color bar is in unit of $\mu \mathrm{m}$.

The approach proposed in this paper has been then extensively checked in the realistic case of noisy field data, obtained by adding a zero-mean complex Gaussian noise to the noise-free data provided with GRASP. Therefore, the noisy samples on the terrestrial framework are given by:

$$
F_{C}\left(\Theta_{h}, \Phi_{k}\right)=F_{C}^{T}\left(\Theta_{h}, \Phi_{k}\right)+n_{R}\left(\Theta_{h}, \Phi_{k}\right)+j n_{I}\left(\Theta_{h}, \Phi_{k}\right)
$$

wherein $F_{C}^{T}$ is the field's "ideal" value and $n_{R}$ and $n_{I}$ are independent Gaussian distributions with mean zero and standard deviation $\sigma=\max _{p, k}\left\{\left|F_{C}^{T}\right|\right\} /$ SNR. This definition of $\sigma$ implies that the SNR is calculated with respect to the maximum of the field pattern.

In Figures 9-12, a displacement of $100 \mu \mathrm{m}$ of the panel under test $P_{i}$ has been retrieved for different values of the
TABLE 1: Recovered deformations on the reflector surface for varying values of the SNR.

\begin{tabular}{lcccc}
\hline $\begin{array}{l}\text { SNR } \\
{[\mathrm{dB}]}\end{array}$ & $\begin{array}{c}\text { Mean in } P_{i} \\
{[\mu \mathrm{m}]}\end{array}$ & $\begin{array}{c}\mid \text { Mean } \mid \text { in } P_{j} \\
{[\mu \mathrm{m}]}\end{array}$ & $\begin{array}{c}\text { RMS } \\
{[\mu \mathrm{m}]}\end{array}$ & Figure \\
\hline 38 & 50 & 70 & 55.4 & - \\
40 & 90 & 43 & 44.6 & Figure 9 \\
42 & 70 & 40 & 34.9 & - \\
44 & 70 & 35 & 27.8 & Figure 10 \\
46 & 74 & 23 & 22.1 & - \\
48 & 84 & 18 & 17.7 & Figure 11 \\
50 & 82 & 13 & 14.3 & - \\
56 & 79 & 8 & 7.5 & Figure 12 \\
62 & 80 & 8 & 4.5 & - \\
$\infty$ & 80 & 5 & 2.8 & Figure 8 \\
\hline
\end{tabular}

SNR. In Table 1, the accuracy of the procedure has been tested by

(i) the comparison between the recovered deformation of $P_{i}$ and that of $P_{j}$, which is the panel with the highest deformation among all the panels of the reflector surface different from $P_{i}$;

(ii) the evaluation of the rms value (RMS in Table 1), over all the reflector panels.

As apparent in Table 1 and Figures 9, 10, 11, and 12, a beam peak voltage SNR in the range $40-44 \mathrm{~dB}$ is enough to achieve an accuracy of $100 \mu \mathrm{m}$.

\section{Conclusion}

The surface profile diagnostic of large reflector antennas has been addressed with a new microwave holographic technique, based on the direct use of Fresnel-field data. The proposed procedure provides the inversion of the Field data by using a SVD regularization technique. A thorough analysis, based on data computed using the well-assessed GRASP commercial software, has shown the accuracy of this holographic technique. The high flexibility of the procedure (e.g., in the choice of the operating frequency and measuring distance) and the stronger signal achievable with a terrestrial transmitter, make this procedure very promising to retrieve the panels misalignments of large reflector antennas and radio telescopes.

\section{Acknowledgment}

The authors would like to thank G. A. Casula and P. Bolli for helpful discussion and suggestions.

\section{References}

[1] M. J. Kesteven, B. F. Parsons, and D. E. Yabsley, "Antenna reflector metrology: the Australia telescope experience," IEEE Transactions on Antennas and Propagation, vol. 36, no. 10, pp. 1481-1484, 1988. 
[2] R. Subrahmanyan, "Photogrammetric measurement of the gravity deformation in a Cassegrain antenna," IEEE Transactions on Antennas and Propagation, vol. 53, no. 8, pp. 25902596, 2005.

[3] Y. Rahmat-Samii, "Surface diagnosis of large reflector antennas using microwave holographic metrology," Radio Science, vol. 19, no. 5, pp. 1205-1217, 1984.

[4] P. F. Scott and M. Ryle, "A rapid method for measuring the figure of a radio telescope reflector," Monthly Notices of the Royal Astronomical Society, vol. 178, pp. 539-545, 1977.

[5] J. C. Bennet, A. P. Anderson, P. A. McInnes, and A. J. T. Whitaker, "Microwave holografic metrology of large reflector antennas," IEEE Transactions on Antennas and Propagation, vol. 24, pp. 295-303, 1976.

[6] Y. Rahmat-Samii, "Microwave holography of large reflector antennas-simulation algorithms," IEEE Transactions on Antennas and Propagation, vol. 33, pp. 1194-1203, 1985.

[7] G. Mazzarella, G. Montisci, N. Schirru, and G. Serra, "A Backus-Gilbert approach to the reflector surface reconstruction," in Proceedings of the IEEE Antennas and Propagation Society International Symposium (APS '06), pp. 1053-1056, July 2006.

[8] B. Nikolic, R. M. Prestage, D. S. Baiser, C. J. Chandler, and R. E. Hills, "Out-of-focus holography at the Green Bank Telescope," Astronomy and Astrophysics, vol. 465, no. 2, pp. 685-693, 2007.

[9] D. J. Rochblatt and Y. Rahmat-Samii, "Effects of measurement errors on microwave antenna holography," IEEE Transactions on Antennas and Propagation, vol. 39, no. 7, pp. 933-942, 1991.

[10] J. W. M. Baars, R. Lucas, J. G. Mangum, and J. A. Lopez-Perez, "Near-field radio holography of large reflector antennas," IEEE Antennas and Propagation Magazine, vol. 49, no. 5, pp. 24-31, 2007.

[11] J. Ruze, "Antenna tolerance theory-a review," Proceedings of the IEEE, vol. 54, no. 4, pp. 633-640, 1966.

[12] C. Hansen, Rank-Deficient and Discrete Ill-Posed Problems, SIAM, New York, NY, USA, 1998.

[13] Y. Rahmat-Samii and J. Lemanczyk, "Application of spherical near-field measurements to microwave holographic diagnosis of antennas," IEEE Transactions on Antennas and Propagation, vol. 36, no. 6, pp. 869-878, 1988.

[14] C. A. Balanis, Antenna Theory, Analysis and Design, John Wiley \& Sons, New York, NY, USA, 1997.

[15] A. C. Ludwig, "The definition of cross-polarization," IEEE Transactions on Antennas and Propagation, vol. 21, pp. 116119, 1973.

[16] M. Bertero, C. de Mol, and E. R. Pike, "Linear inverse problems with discrete data. I. General formulation and singular system analysis," Inverse Problems, vol. 1, no. 4, article 004, pp. 301330, 1985.

[17] M. Bertero, C. de Mol, and E. R. Pike, "Linear inverse problems with discrete data: II. Stability and regularisation," Inverse Problems, vol. 4, no. 3, article 004, pp. 573-594, 1988.

[18] P. Bolli, G. Mazzarella, G. Montisci, and G. Serra, "An alternative solution for the reflector surface retrieval problem," Progress in Electromagnetics Research, vol. 82, pp. 167-188, 2008.

[19] O. M. Bucci and G. di Massa, "Truncation error in the application of sampling series to electromagnetic problems," IEEE Transactions on Antennas and Propagation, vol. 36, no. 7, pp. 941-949, 1988.

[20] O. M. Bucci and G. Franceschetti, "On the spatial bandwidth of scattered fields," IEEE Transactions on Antennas and Propagation, vol. 35, no. 12, pp. 1445-1455, 1987.

[21] D. C. Ghiglia and M. D. Pritt, Two Dimensional Phase Unwrapping: Theory, Algorithms and Software, John Wiley \& Sons, New York, NY, USA, 1998. 

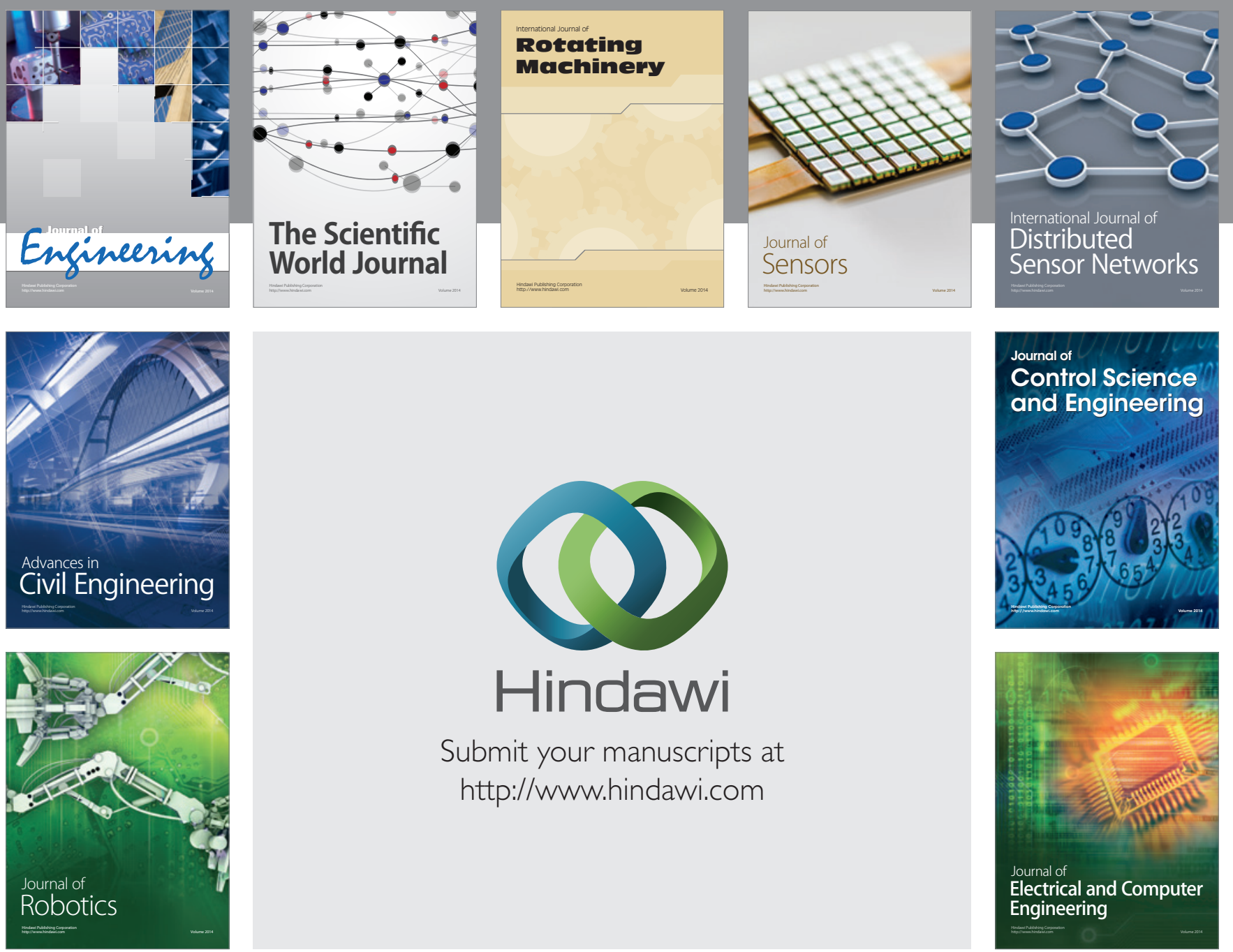

Submit your manuscripts at

http://www.hindawi.com
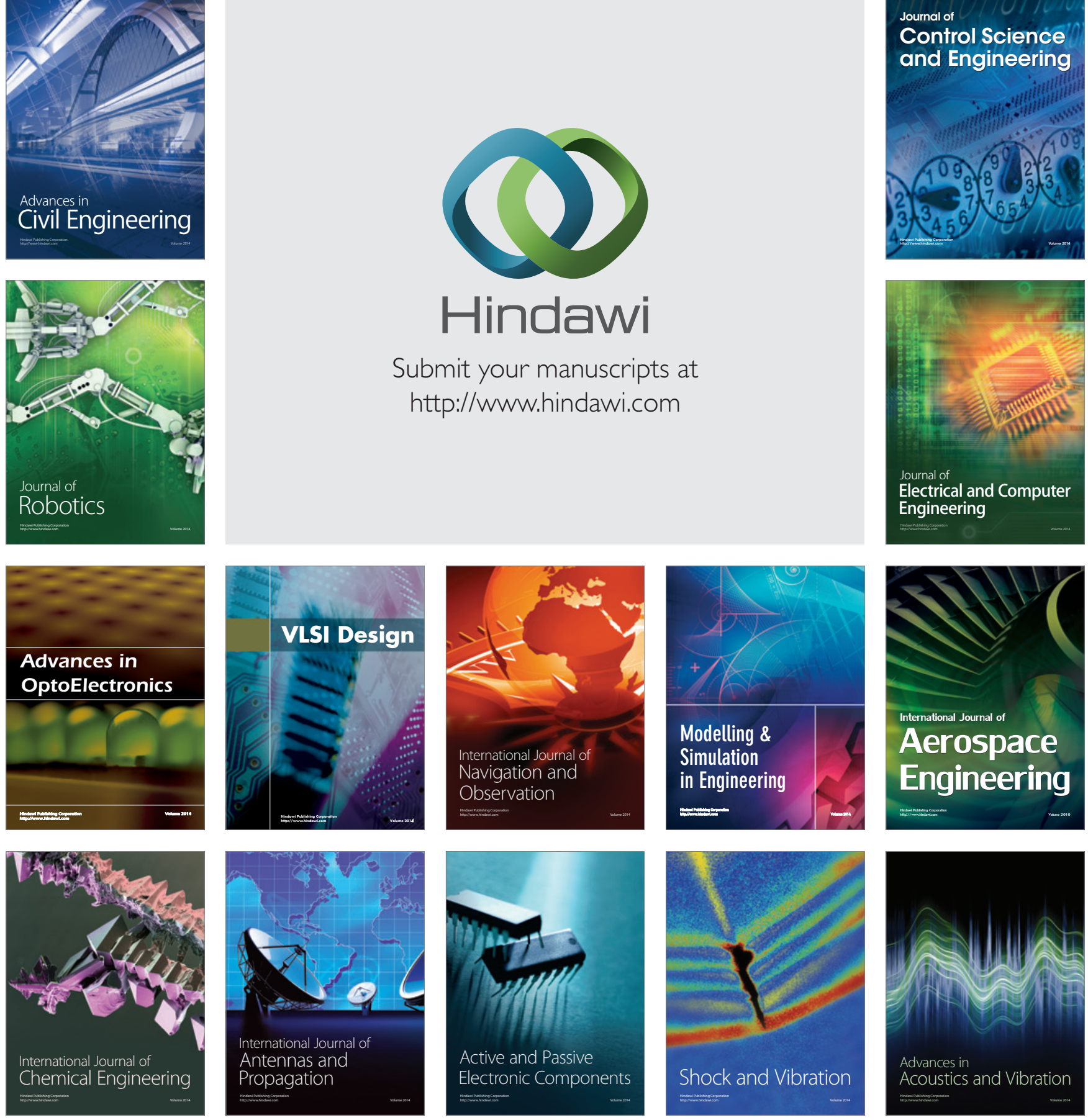